\title{
Circulating microparticle subpopulation in metabolic syndrome: relation to oxidative stress and coagulation markers
}

\author{
Asmaa M Zahran' \\ Sohair K Sayed ${ }^{2}$ \\ Heba A Abd El Hafeez ${ }^{2}$ \\ Walaa A Khalifa ${ }^{3}$ \\ Nahed A Mohamed ${ }^{4}$ \\ Helal F Hetta ${ }^{5,6}$ \\ 'Department of Clinical Pathology, \\ South Egypt Cancer Institute, Assiut, \\ Egypt; '2Department of Clinical \\ Pathology, Faculty of Medicine, Assiut \\ University, Assiut, Egypt; ${ }^{3}$ Department \\ of Internal Medicine, Faculty of \\ Medicine, Assiut University, Assiut, \\ Egypt; ${ }^{4}$ Department of Medical \\ Biochemistry, Faculty of Medicine, \\ Assiut University, Assiut, Egypt; \\ ${ }^{5}$ Department of Medical Microbiology \\ and Immunology, Faculty of Medicine, \\ Assiut University, Assiut, Egypt; \\ ${ }^{6}$ Department of Internal Medicine, \\ University of Cincinnati College of \\ Medicine, Cincinnati, OH, USA
}

Background: Circulating microparticles (MPs) contribute to the pathogenesis of atherothrombotic disorders and are raised in cardiovascular diseases. Herein, we aimed to investigate the effect of moderate metabolic abnormalities in an early stage of metabolic syndrome (MetS) on the level of MP subpopulations and to study relationships between MP subpopulations and both oxidative stress and coagulation markers.

Methods: Flow cytometry used to evaluate circulating MPs subpopulations in 40 patients with an early stage MetS and 30 healthy controls. ELISA was used to quantify plasminogen activator inhibitor type 1/tissue plasminogen activator (PAI-1/TPA) while plasma glutathione peroxidase (GPx) activity was measured spectrophotometrically.

Results: Total MPs were significantly elevated in MetS $(P<0.001)$. Glutathione peroxidase and PAI1/TPA activity was significantly increased in subjects with MetS $(P<0.001)$. Waist circumference, diastolic blood pressure, and total cholesterol positively influenced levels of total MPs, platelet-derived microparticles, and endothelium-derived microparticles. Fasting blood glucose, cholesterol, triglycerides, and low-density lipoprotein positively influenced the coagulation factors (TPA, PAI1). However, high-density lipoprotein negatively influenced platelet-derived MPs and factors associated with fibrinolysis (TPA, PAI1).

Conclusion: Elevated circulating MPs are associated with MetS abnormalities, oxidative stress and coagulation factors and may act as early predictor of metabolic syndrome with risk of cardiovascular disease.

Keywords: circulating microparticles, metabolic syndrome, oxidative stress, coagulation, CVD

\section{Introduction}

Metabolic syndrome (MetS) is a group of cardiometabolic abnormalities comprising abdominal obesity, hypertension, hyperglycemia, and dyslipidemia, and associates with other comorbidities, including prothrombotic and proinflammatory states. ${ }^{1}$ Insulin resistance, fatty acids, and increased proinflammatory cytokines produced by adipocytes and macrophages are considered the main risk factors in MetS pathophysiology.2,3

Microparticles (MPs) are small, intact, membranous vesicular structures, lack nuclei, express phosphatidylserine on the surface, and range in size from 0.1 to 1 $\mu \mathrm{m} .{ }^{4}$ Nearly all cell types can release MPs: they originate from plasma membranes of endothelial cells, vascular smooth-muscle cells, leukocytes, platelets, erythrocytes, epithelial cells, and tumor cell lines. ${ }^{5}$ Endothelium-derived MPs (EMPs) are a biological marker of endothelial injury and vascular tone disorders. ${ }^{6}$ EMPs derive from activated or apoptotic endothelial cells, and may play an important role in vascular rebuild and endothelial repair. ${ }^{7}$ Additionally, circulating EMPs derived from activated
Correspondence: Helal F Hetta Department of Internal Medicine, University of Cincinnati College of Medicine, 23I Albert B Sabin Way, PO Box 670595, Cincinnati, OH 45267-0595, USA

$\mathrm{Tel}+15135586110$

$\mathrm{Fax}+|5| 35581744$

Email helal.hetta@uc.edu 
endothelial cells have been shown to have proangiogenic and cardioprotective properties. ${ }^{8}$ In contrast, apoptotic EMPs are considered markers of endothelial cell injury and vascular aging. ${ }^{1}$ Platelet-derived MPs (PMPs) are the most abundant subtype measured in human plasma. ${ }^{9}$ PMPs can mediate multiple cellular responses, predominantly affecting protein and lipid metabolism, coagulation, and inflammation. ${ }^{10}$

Oxidative stress plays a considerable role in the pathogenesis of MetS components and insulin resistance. ${ }^{11}$ The main antioxidant enzymes are glutathione peroxidase (GPx), superoxide dismutase, catalase, and myeloperoxidase. ${ }^{12}$ Peroxidases act as preventive antioxidants to detoxify damaged lipid peroxides or other peroxides from blood and organic substrates. On the other hand, these enzymes function to start oxidative reactions, thereby generating a source of reactive oxygen species (ROS). ${ }^{13}$

PAI1 is the main enzyme inhibitor of the plasminogenactivator system, and increased expression of PAI1 suppresses endogenous fibrinolysis, leading to fibrin accumulation in basement membranes and interstitial tissues. ${ }^{14-16}$ Remarkably, the predictive ability of PAI1 disappears after adjustment for markers of MetS, such as body-mass index (BMI), triglycerides (TGs), and high-density-lipoprotein cholesterol (HDL-C), suggesting that MetS is a precondition for high plasma-PAI1 levels in patients prone to atherothrombosis. Therefore, high plasma concentrations of PAI1 and TPA reflect a state of fibrinolytic dysfunction that increases the propensity to develop arterial thrombosis and in turn may increase cardiovascular disease (CVD) in people with MetS. ${ }^{17}$

Although MPs are a biomarker of vascular dysfunction in MetS patients, their significance in MetS patients with congestive heart failure remains controversial. An example of this controversy is that it is still unknown if circulating MPs found in peripheral blood cause injury of the endothelium and worsening of congestive heart failure. ${ }^{18}$ As such, in this study we aimed to investigate whether moderate metabolic abnormalities in an early stage of MetS may alter the level of MP subpopulations and to assess the relationship between MP subpopulations and oxidative stress (plasma GPx activity) and coagulation markers (PAI1 and TPA).

\section{Methods}

A case-control study was conducted between May 2015 and May 2017 in the Department of Internal Medicine, Assiut University Hospital, Assiut, Egypt. The study was approved by the ethical committee of the Faculty of Medicine, Assiut University, Assiut, Egypt, according to the code of ethics of the World Medical Association (Declaration of Helsinki).
Written informed consent was obtained from each participant after explaining the study to them.

Two groups were included in our study; the first group had 40 patients with MetS, which is defined by the presence of three of the following components: fasting glucose $>5.5 \mathrm{mmol} / \mathrm{L}$, triglyceride $\geq 1.65 \mathrm{mmol} / \mathrm{L}, \mathrm{HDL}-\mathrm{C}<1.0 \mathrm{mmol} / \mathrm{L}$ (men) or $<1.3$ $\mathrm{mmol} / \mathrm{L}$ (women), raised blood pressure (BP): systolic pressure $\geq 130 \mathrm{and} /$ or diastolic $\geq 85 \mathrm{~mm} \mathrm{Hg}$, and waist circumference $>102 \mathrm{~cm}$ (men) or $>88 \mathrm{~cm}$ (women). The criteria used were based on the definition of the National Cholesterol Education Program Adult Treatment Panel III (NCEP ATP-III), 2001. ${ }^{19}$ A total of 14 MetS patients out of 40 (35\%) were taking antihypertensive treatment. Patients with a history of CVD, chronic inflammatory disease, and cancer or receiving an oral antidiabetic treatment or insulin were excluded. The second group included 30 healthy controls (19 women, 11 men) recruited from staff of the hospital that did not have any known medical disorder or were taking any prescribed medication and who met none of the MetS criteria.

\section{Measurement of blood pressure and anthropometric indices}

BP measurements were taken from each subject's right arm in the seated position using a sphygmomanometer after 10 minutes of rest in a quiet room in the morning. Three successive BP readings were obtained at 5-minute intervals and averaged. Height and body weight were measured in the morning without shoes in a fasting state, and BMI was computed as weight in kilograms divided by height in meters squared. WC was measured midway between the lower-rib margin and the superior iliac spine at the end of gentle expiration in a standing position.

\section{Biochemical analysis}

Laboratory investigations were done in the Department of Clinical Pathology of Assiut University Hospital and the South Egypt Cancer Institute. Venous blood samples $(10 \mathrm{~mL})$ were obtained from all subjects in the fasting state (8-10 am) after 15 minutes' rest, because of the diurnal variation of plasma PAI1. ${ }^{47}$ Collected blood was divided immediately: $2 \mathrm{~mL}$ in EDTA tubes for complete blood count and GPx activity, $3.5 \mathrm{~mL}$ in $3.2 \%$ $\mathrm{Na}$ citrate for MP isolation, $2 \mathrm{~mL}$ in $3.2 \% \mathrm{Na}$ citrate for PAI2 + TPA detection, and the rest in plain tubes without any additives. After 20 minutes of incubation at room temperature, plain tubes were centrifuged for 10 minutes at 1,200 $\mathrm{g}$. Sera were separated carefully and used for glucose, lipoprotein, urea, and creatinine detection. Plasma samples for PAI2 + TPA and GPX activity were aliquoted and stored $\left(-80^{\circ} \mathrm{C}\right)$ until required for analysis. Parameters determined in routine laboratory testing 
were platelets, total leukocyte count, and hemoglobin levels, determined using a Sysmex XE-5000 automated hematology system. A Cobas 6,000 analyzer (Roche) was used to determine concentrations of glucose, TGs, total cholesterol, low-density lipoprotein (LDL) cholesterol, and HDL-C.

\section{GPx activity and PAII/TPA detection}

GPx activity was measured using spectrophotometry (BioDiagnostics, Upton-upon-Severn, UK). Determination of PAI1/TPA was done using a human PAI1/TPA ELISA kit (ab108892; Abcam, Cambridge, UK).

\section{Microparticle isolation and characterization}

Citrate blood samples were used to isolate MPs. Cells were removed by centrifugation at $1550 \mathrm{~g}$ at $20^{\circ} \mathrm{C}$ for 20 minutes. Then, $250 \mu \mathrm{L}$ plasma was centrifuged twice at 18,000 $\mathrm{g}$ at $20^{\circ} \mathrm{C}$ for 30 minutes. The supernatant was removed and the pellet suspended in PBS. MPs samples $(5 \mu \mathrm{L})$ were diluted in PBS containing $\mathrm{CaCl}_{2}$ and incubated for 20 minutes at room temperature with $5 \mu \mathrm{L}$ fluorescein isothiocyanate-conjugated annexin V (IQ Products, Groningen, The Netherlands), PerCPconjugated CD41, APC-conjugated CD45, and phycoerythrin CD144 (BD Biosciences, San Jose, CA, USA). FACSCalibur

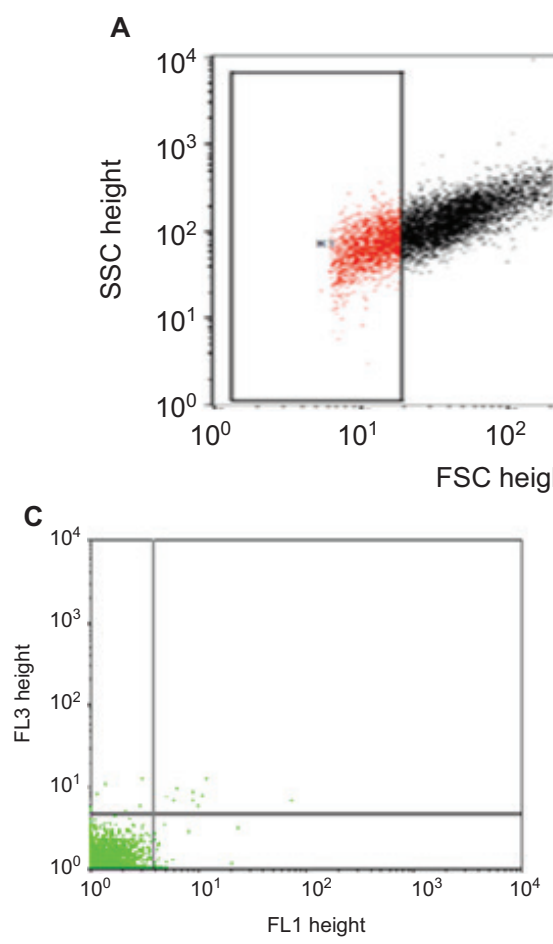

flow cytometry with CellQuest software (BD Biosciences) was used to analyze 50,000 events of MPs. Antihuman IgG isotype-matched negative control was used with each sample.

MPs were identified by comparing their size with that of calibrated reference beads of $1 \mu \mathrm{m}$ (latex beads, aminemodified polystyrene, fluorescent red aqueous suspension, 1 $\mu \mathrm{m}$ mean particle size; Sigma-Aldrich, St Louis, MO, USA) and by their positivity for annexin V. Total MPs (TMPs) are expressed as a percentage of total events. EMPs were defined as $\mathrm{CD} 45^{-} \mathrm{CD} 144^{+} \mathrm{MPs}$ and PMPs as CD41 ${ }^{+}$MPs. PMPs and EMPs are expressed as percentage of TMPs (Figure 1).

\section{Statistical analysis}

Data were tested for normality using the Anderson-Darling test and for homogeneity variance prior to further statistical analysis. Categorical variables are given as number and percentage and continuous variables as means \pm SD for normally distributed data, while medians and IQR are used for abnormally distributed data. Fisher's exact test was used to compare between categorical variables. Comparison between continuous variables was done by $t$-test (parametric) and Mann-Whitney $U$ test (nonparametric). Pearson's correlation coefficient was used to assess correlations among continuous data. Multiple linear regressions were used to detect signifi-
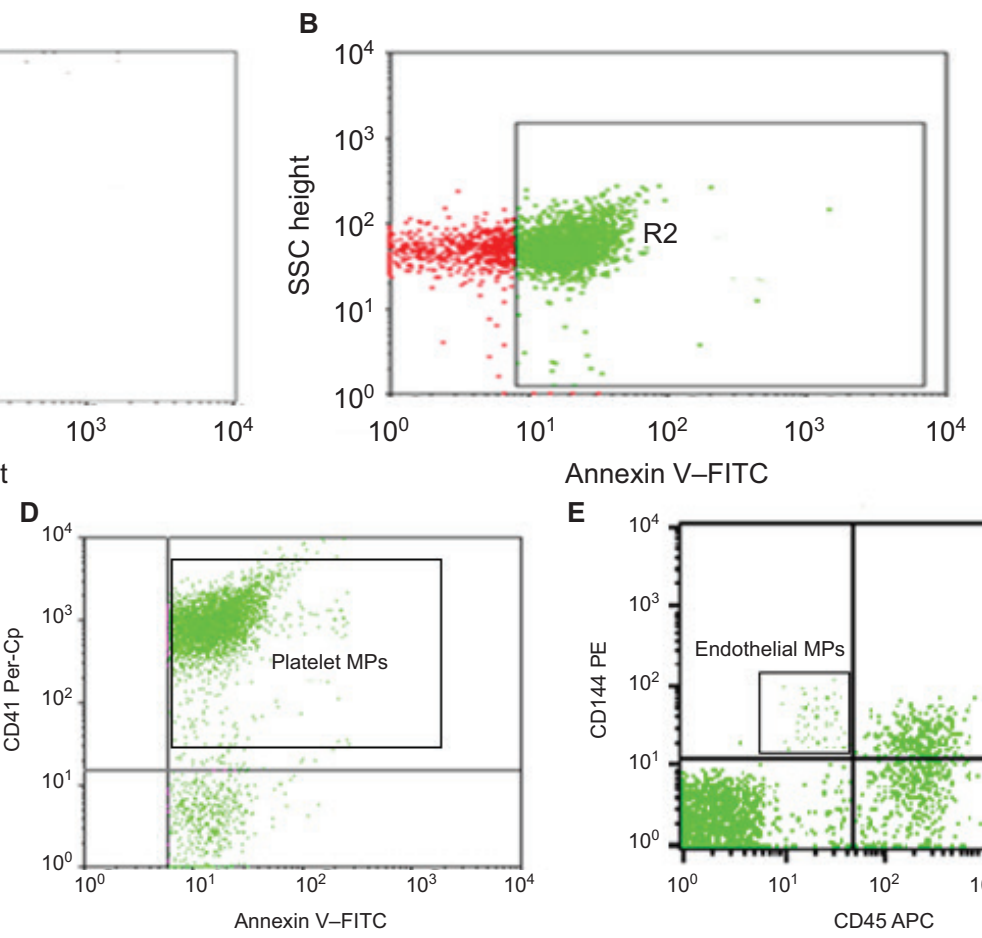

E

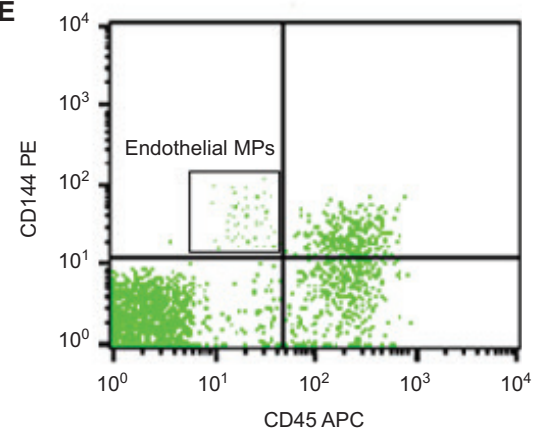

Figure I Flow-cytometry analysis of circulating microparticles (MPs).

Notes: (A) FSC and SSC histogram defining MPs according to size (RI). (B) Events defined as MPs (RI) were selected for their annexin $V$ binding (R2). (C) Negative-isotype control. (D, E) Annexin V-positive MPs (total MPs) were further examined for expression of cell-specific antibodies - CD45, CDI44, and CD4I antibodies - to detect endothelium and platelet MPs.

Abbreviations: FSC, forward scatter; SSC, side scatter; FITC, fluorescein isothiocyanate. 
cant predictors. Two-tailed $P<0.05$ was considered statistically significant. All analyses were performed with SPSS 20.0.

\section{Results}

\section{General characterization of study groups}

The study included 40 participants with MetS (28 males and 12 females) and 30 healthy controls. Table 1 shows basic clinical and metabolic characteristics of the subjects. MetS was more common in females than males $(70.0 \%$ vs $30 \%$, respectively). For biochemical analysis, there were significant differences in WC, weight, BMI, FBG, cholesterol, TG, LDL, and HDL means when compared to the control group $(P<0.001$ for all). Also, GPx activity and PAT-1/TPA levels were significantly higher in patients with MetS than the healthy control group $(P<0.001$ and $P=0.001$, respectively).

\section{Frequency of MP subpopulations among MetS patients}

We found that patients with MetS had significantly higher frequencies of TMPs, PMPs $(P<0.001)$, and EMPs
$(P=0.003)$ in comparison to healthy controls, as shown in Figure 2.

\section{Association between frequency of MP subpopulations, plasma GPx, and PAII/ TPA with disease parameters}

The frequency of TMPs was positively correlated with WC, BMI, FBG, systolic BP (SBP), diastolic BP (DBP), TGs, and cholesterol and LDL with TMPs, PMPs, and EMPs. Cholesterol, LDL, and BMI were positively correlated with TMPs, PMPs, and EMPs, as shown in Table 2.

\section{Correlations among plasma levels of GPx and PAII/TPA, MP subpopulations, and MetS clinical parameters}

Table 3 shows associations among plasma levels of GPx activity, PAI1/TPA, and frequency of MP subpopulations. The frequency of TMPs and EMPs was positively correlated with both GPx activity and PAI1/TPA. However, the frequency of PMPs was positively correlated with GPx, but not with PAI1/

Table I Baseline clinical and laboratory characteristics of MetS patients and healthy controls

\begin{tabular}{|c|c|c|c|}
\hline & Cases (total 40), n (\%) & Controls (total 30), n (\%) & $P$-value \\
\hline Age (years) & $44.4 \pm 12.2$ & $39.1 \pm 11.7$ & 0.071 \\
\hline Male:female, n (\%) & $12(30 \%): 28(70 \%)$ & II (36.7\%): 19 (63.3\%) & 0.557 \\
\hline WC (cm) & $111.03 \pm 11.04$ & $67.47 \pm 13.19$ & $<0.001$ \\
\hline Weight (kg) & $103.28 \pm 11.58$ & $63.33 \pm 6.64$ & $<0.001$ \\
\hline BMI $\left(\mathrm{kg} / \mathrm{m}^{2}\right)$ & $37.78 \pm 3.82$ & $23.1 \pm 1.12$ & $<0.001$ \\
\hline SBP (mmHg) & $132.23 \pm 16.09$ & $112.67 \pm 9.07$ & $<0.001$ \\
\hline DBP (mmHg) & $86 \pm 8.93$ & $76.67 \pm 6.61$ & $<0.001$ \\
\hline FBG (mmol/L) & $5.76 \pm 1.17$ & $4.56 \pm 0.61$ & $<0.001$ \\
\hline Cholesterol (mmol/L) & $5.13 \pm 0.98$ & $3.7 \pm 0.67$ & $<0.001$ \\
\hline TGs (mmol/L) & $1.84 \pm 1.13$ & $0.77 \pm 0.3$ & $<0.001$ \\
\hline HDL (mmol/L) & $\mathrm{I} .17 \pm 0.24$ & $1.31 \pm 0.19$ & 0.014 \\
\hline LDL (mmol/L) & $3.1 \pm 0.91$ & $2.04 \pm 0.58$ & $<0.001$ \\
\hline Urea (mmol/L) & $4.07 \pm 1.22$ & $4.35 \pm 1.05$ & 0.322 \\
\hline Creatinine ( $\mu \mathrm{mol} / \mathrm{L})$ & $75.28 \pm|4.5|$ & $68.29 \pm 21.33$ & 0.107 \\
\hline WBCs $\left(10^{9} / L\right)$ & $6.1 \pm 1.67$ & $6.39 \pm 1.57$ & 0.458 \\
\hline $\mathrm{Hb}(\mathrm{g} / \mathrm{dL})$ & $12.78 \pm 0.99$ & $13.37 \pm 1.11$ & 0.022 \\
\hline Platelets (109/L) & $261.63 \pm 82.96$ & $274.9 \pm 53.09$ & 0.446 \\
\hline GPx activity $(\mathrm{mU} / \mathrm{mL})$ & $2.98 \pm 1.61$ & $1.54 \pm 1.33$ & $<0.001$ \\
\hline PAII/TPA (ng/mL) & $4.22 \pm 3.41$ & $1.94 \pm 0.7$ & 0.001 \\
\hline \multicolumn{4}{|l|}{ MetS diagnostic criteria } \\
\hline WC & $87.5 \%$ & NA & NA \\
\hline $\mathrm{BP}$ & $67.5 \%$ & NA & \\
\hline Fasting glycemia & $50 \%$ & NA & \\
\hline HDL cholesterol & $22.5 \%$ & NA & \\
\hline TGs & $60 \%$ & NA & \\
\hline
\end{tabular}

Notes: There were significant differences in means of WC, weight, BMI, FBG, cholesterol, TGs, LDL, and HDL when compared to the control group ( $P<0.00 \mathrm{I}$ for all). Also, GPx enzyme activity and PAII/TPA levels were significantly higher in MetS patients than the healthy control group $\left(P<0.00 \mathrm{I}\right.$ and $P=0.00 \mathrm{I}$, respectively). ${ }^{\mathrm{a}} \mathrm{Mann}-\mathrm{Whitney} U$ test, $P<0.05$ significant. Data expressed as means \pm SD.

Abbreviations: MetS, metabolic syndrome; WC, waist circumference; BMI, body-mass index; FBG, fasting blood glucose; TGs, triglycerides; HDL, high-density lipoprotein; LDL, low-density lipoprotein; SBP, systolic blood pressure; DBP, diastolic BP; GPx, glutathione peroxidase; WBCs, white blood cells; Hb, hemoglobin. 
TPA. Furthermore, plasma GPx was positively correlated with WC, BMI, FBG, SBP, DBP, LDL, cholesterol, and TGs but not HDL, platelets, or white blood cells. However, PAI1/ TPA showed positive correlations with SBP, cholesterol, TGs, and LDL.

\section{Multivariate-regression analysis of influence of MetS parameters on MP subpopulation and activity of GPx and PAII/TPA}

Multivariate regression-analysis results on the influence of MetS parameters on the MP subpopulation, activity of GPx, and PAI1/TPA are shown in Table 4. Regarding MP subpopulations, WC positively influenced levels of TMPs. DBP positively influenced levels of EMPs. Cholesterol positively influenced levels of TMPs, PMPs, and EMPs. FBG, cholesterol, TGs, and LDL positively influenced coagulation factors (TPA and PAI1). However, HDL negatively influenced PMPs and coagulation factors.

\section{Discussion}

MetS is a serious health problem where a cluster of conditions, including increased BP, high blood sugar, excess body fat around the waist, and abnormal cholesterol or TG levels, occur together. ${ }^{20}$ In our study, fasting hyperglycemia was present in $50 \%$ of our studied patients, as insulin resistance plays a major pathogenic role in the development of MetS.

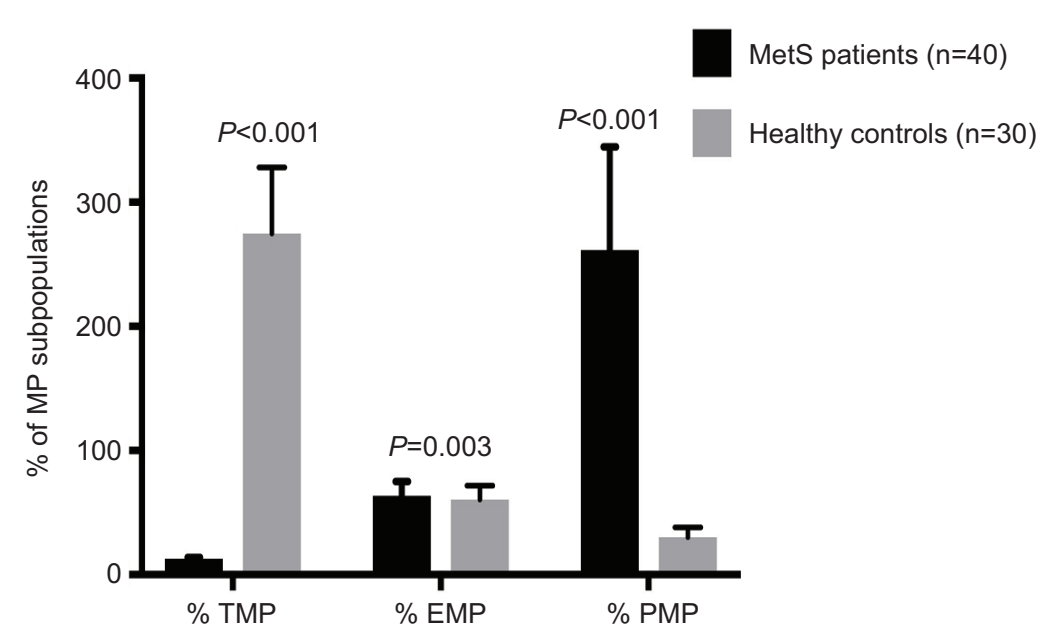

Figure 2 Differences in percentages of MP subpopulations between 40 MetS and 30 healthy controls.

Notes: There were highly significant differences in percentages of TMPs $(P<0.00 \mathrm{I})$, PMPs $(P<0.00 \mathrm{I})$, and EMPs $(P=0.003)$ between the two groups.

Abbreviations: MP, microparticle; MetS, metabolic syndrome; TMP, total MP; PMP, platelet-derived MP; EMP, endothelium-derived MP.

Table 2 Spearman correlations between percentages of MP subpopulations for clinical parameters in the MetS group

\begin{tabular}{|c|c|c|c|c|c|c|}
\hline & \multicolumn{2}{|c|}{ \%TMPs } & \multicolumn{2}{|c|}{ \%PMPs } & \multicolumn{2}{|c|}{ \%EMPs } \\
\hline & $r$ & $P$-value & $r$ & $P$-value & $r$ & $P$-value \\
\hline WC & 0.40 & 0.010 & 0.46 & 0.003 & 0.37 & 0.018 \\
\hline BMI & 0.46 & 0.003 & 0.46 & 0.003 & 0.40 & 0.011 \\
\hline SBP & 0.96 & $<0.001$ & 0.94 & $<0.001$ & 0.94 & $<0.001$ \\
\hline DBP & 0.54 & $<0.001$ & 0.62 & $<0.001$ & 0.42 & 0.008 \\
\hline Cholesterol & 0.97 & $<0.001$ & 0.96 & $<0.001$ & 0.95 & $<0.001$ \\
\hline TGs & 0.48 & 0.002 & 0.44 & 0.005 & 0.50 & 0.001 \\
\hline HDL & 0.05 & 0.758 & -0.01 & 0.963 & 0.08 & 0.642 \\
\hline LDL & 0.81 & $<0.001$ & 0.79 & $<0.001$ & 0.81 & $<0.001$ \\
\hline FBG & 0.36 & 0.021 & 0.40 & 0.011 & 0.33 & 0.037 \\
\hline WBCs & 0.13 & 0.416 & 0.16 & 0.329 & 0.09 & 0.575 \\
\hline Hemoglobin & -0.01 & 0.956 & 0.04 & 0.790 & -0.04 & 0.820 \\
\hline Platelets & 0.03 & 0.831 & 0.02 & 0.894 & 0.06 & 0.695 \\
\hline
\end{tabular}

Abbreviations: MP, microparticle; MetS, metabolic syndrome; WC, waist circumference; BMI, body-mass index; FBG, fasting blood glucose; TGs, triglycerides; HDL, highdensity lipoprotein; LDL, low-density lipoprotein; SBP, systolic blood pressure; DBP, diastolic BP; WBCs, white blood cells; TMPs, total MPs; PMPs, platelet MPs; EMPs, endothelial MPs. 
Helal et al found that the percentage of fasting hyperglycemia was $79.5 \% .{ }^{23}$ On the other hand, Zaki et al found fasting hyperglycemia in $15 \%$ of studied individuals. ${ }^{24}$ Other studies have found that the prevalence of fasting hyperglycemia was much lower $(8.3 \%) .{ }^{25,26}$ These differences in results can be explained by differences in age, dietary habits, and genetic factors. Hypertension was found in $67.5 \%$ of MetS individuals. Similar results were found by Helal et al, who detected high BP in $70.5 \%$ of MetS individuals. ${ }^{23}$

With regard to lipid profile, we detected low HDL $22.5 \%$; however, hypertriglyceridemia was present in $60 \%$

Table 3 Spearman correlations among plasma levels of GPx, PAII/TPA, MP subpopulations, and MetS clinical parameters in the MetS group

\begin{tabular}{|c|c|c|c|c|}
\hline & \multicolumn{2}{|l|}{ GPx } & \multicolumn{2}{|c|}{ PAII/TPA } \\
\hline & $r$ & $P$-value & $r$ & $P$-value \\
\hline \%TMPs & 0.97 & $<0.001$ & 0.31 & 0.04 \\
\hline \%EMPs & 0.95 & $<0.001$ & 0.43 & 0.006 \\
\hline \%PMPs & 0.99 & $<0.001$ & 0.23 & 0.14 \\
\hline WC & 0.46 & 0.003 & 0.29 & 0.073 \\
\hline BMI & 0.46 & 0.003 & 0.14 & 0.40 \\
\hline SBP & 0.96 & $<0.001$ & 0.31 & 0.05 \\
\hline DBP & 0.59 & $<0.001$ & -0.09 & 0.59 \\
\hline Cholesterol & 0.98 & $<0.001$ & 0.35 & 0.02 \\
\hline TGs & 0.44 & 0.004 & 0.32 & 0.04 \\
\hline HDL & 0.02 & 0.91 & 0.06 & 0.70 \\
\hline LDL & 0.82 & $<0.001$ & 0.32 & 0.04 \\
\hline FBG & 0.39 & 0.013 & 0.06 & 0.735 \\
\hline WBCs & 0.19 & 0.250 & -0.11 & 0.486 \\
\hline Hemoglobin & 0.02 & 0.891 & -0.09 & 0.585 \\
\hline Platelets & 0.09 & 0.564 & 0.14 & 0.403 \\
\hline
\end{tabular}

Abbreviations: MP, microparticle; MetS, metabolic syndrome; WC, waist circumference; BMI, body-mass index; FBG, fasting blood glucose; TGs, triglycerides; HDL, high-density lipoprotein; LDL, low-density lipoprotein; SBP, systolic blood pressure; DBP, diastolic BP; GPx, glutathione peroxidase; TMPs, total MPs; PMP, platelet MPs; EMPs, endothelial MPs. of MetS subjects. Eshtiaghi et al also showed that low HDL and elevated TGs were significant predictors of metabolic health conditions. ${ }^{27}$ On the other hand, hypertriglyceridemia was present in $23 \%$ of individuals in the study of Zaki et al. ${ }^{24}$ This may be explained by differences in patient characteristics in different studies.

Concerning the frequency of circulating MP subpopulations, we found that the frequency of TMPs, PMPs, and EMPs was elevated in subjects with MetS compared to healthy subjects. From these data, we can hypothesize that these MPs were produced in response to MetS. Agouni et al reported that patients with different types of MetS might have different patterns of MPs. ${ }^{28}$ Because of the procoagulant properties of annexin $\mathrm{V}^{+} \mathrm{MPs}$, they may participate in fibrinolysis impairment and thrombinogenesis elevation.

In addition to TMPs, we found an increase in the frequency of PMPs in MetS subjects. Similar findings have been found by previous studies. ${ }^{23,29}$ In contrary to our findings, Arteaga et al did not find a significant change in PMPs, although they did find increased platelet activation in MetS subjects. ${ }^{30}$ The difference in results may be explained by the markers used for determination of PMPs (CD31/CD42b from Arteaga et al vs CD41 in our study). Also, we found an increase in EMPs in MetS subjects. Although there have been few studies to identify MPs in patients with MetS, Arteaga et al reported that EMPs were elevated and that this resulted from endothelial cell apoptosis, but not activation. EMPs can also aggravate endothelial dysfunction through loss of endothelium-dependent flow-mediated vasodilatation, but the mechanism involved has not been analyzed yet. ${ }^{28}$

We studied the association between MP subpopulations and disease parameters, and interestingly we report positive correlations among EMPs, PMPs, TMPs, and

Table 4 Multiple linear regression to assess predictors of the different types of MPs, GPx activity, and PAII/TPA in the MetS group

\begin{tabular}{|c|c|c|c|c|c|c|c|c|c|c|}
\hline & \multicolumn{2}{|c|}{ TMPs } & \multicolumn{2}{|l|}{ PMPs } & \multicolumn{2}{|c|}{ EMPs } & \multicolumn{2}{|l|}{ GPx } & \multicolumn{2}{|c|}{ PAII/TPA } \\
\hline & $t$ & $P$-value & $t$ & $P$-value & $t$ & $P$-value & $t$ & $P$-value & $t$ & $P$-value \\
\hline WC & 2.15 & 0.041 & 0.64 & 0.526 & 1.89 & 0.071 & 1.25 & 0.230 & 1.86 & 0.082 \\
\hline BMI & 0.16 & $0.87 I$ & 0.09 & 0.932 & 0.12 & 0.902 & 0.33 & 0.743 & 2.45 & 0.027 \\
\hline SBP & 0.82 & 0.422 & 0.55 & 0.587 & 1.69 & 0.103 & 0.93 & 0.366 & 0.18 & 0.857 \\
\hline DBP & 0.62 & 0.539 & 1.76 & 0.091 & 2.88 & 0.008 & 0.62 & 0.543 & 0.14 & 0.891 \\
\hline FBG & 1.48 & 0.151 & 1.56 & 0.131 & 0.59 & 0.563 & 0.65 & 0.524 & 2.81 & 0.013 \\
\hline Cholesterol & 2.93 & 0.007 & 3.70 & 0.001 & 2.35 & 0.027 & -0.42 & 0.682 & 3.99 & 0.001 \\
\hline TGs & 1.86 & 0.075 & 1.05 & 0.303 & 1.18 & 0.249 & 0.98 & 0.342 & 3.23 & 0.006 \\
\hline HDL & 0.77 & 0.449 & -0.88 & 0.386 & 0.44 & 0.663 & 0.39 & 0.705 & 3.66 & 0.002 \\
\hline LDL & 0.72 & 0.477 & 0.38 & 0.705 & 0.95 & 0.350 & 0.24 & 0.816 & 3.89 & 0.001 \\
\hline
\end{tabular}

Abbreviations: MPs, microparticles; MetS, metabolic syndrome; WC, waist circumference; BMI, body-mass index; FBG, fasting blood glucose; TGs, triglycerides; HDL, high-density lipoprotein; LDL, low-density lipoprotein; SBP, systolic blood pressure; DBP, diastolic BP; GPx, glutathione peroxidase; TMPs, total MPs; PMPs, platelet MPs; EMPs, endothelial MPs. 
some MetS risk factors. Circulating MPs could be considered a possible biomarker for identification of subjects with MetS at risk of developing $\mathrm{CV}$ complications or type 2 diabetes.

We found that TMP, EMP, and PMP frequency was positively correlated with WC. These observations point to visceral fat stores playing a possibly harmful role in MP overproduction, a hypothesis that remains to be confirmed. Adipose-tissue inflammation is considered one of the most important pathological processes in obese people without metabolic complications via activation of the NFKB pathway, which results in secretion of inflammatory cytokines and altering endothelial function. ${ }^{31}$ Moreover, we found that $\mathrm{WC}$ is an independent risk factor for TMPs. Our results are in accordance with previous studies suggesting that $\mathrm{WC}$ was a better predictor of metabolic risk factors for developing MetS than BMI, and could be considered a risk factor for diabetes and CVD. ${ }^{32,33}$ Furthermore, our results were also in accordance with previous studies by Goichot et al and Stepanian et al, who showed an association between MPs and obesity risk factors in obese subjects devoid of any CV risk factor. ${ }^{34,35}$

We found that patient blood-glucose levels were associated with levels of MPs, which suggested that TMPs, PMPs, or EMPs could be possible biomarkers for the identification of patients predisposed to type 2 diabetes. ${ }^{36}$ Also, we detect a positive correlation between EMPs and BP. TMPs, EMPs, and PMPs were also positively correlated with fasting hypertriglyceridemia. However, independent associations of TMPs, PMPs, and EMPs with TGs, HDL, and FBG were not found, while associations of increased cholesterol with MPs and increased BP with EMPs were shown. Our results were in accordance with previous studies reporting that dyslipidemia in MetS patients may negatively affect the ability of the endothelium to produce activated microvesicles with angiogenic capacity and secrete apoptosis-derived MPs..$^{37,38}$ The lack of association between MPs and glucose impairment can be explained by the overproduction of inflammatory cytokines and the presence of lipid abnormalities. ${ }^{1}$

We found that GPx activity in erythrocytes in MetS was significantly increased when compared with the control group. These results seem to be related to the early stage of MetS, because subjects with MetS might be under high oxidative stress, antioxidant enzymes are the first line of defense against ROS, and may increase to adjust to higher levels of oxidative stress, which has been supported by previous studies. ${ }^{39,40}$ On the other hand, Ozata et al studied the activity of GPx in the erythrocytes of obese patients and showed a reduction in activity of these enzymes in the blood component and an increase in specific tissue types. ${ }^{41}$ These conflicting results from different studies could be explained by differences in patient characteristics. In the present study, subjects were adults fulfilling early-stage MetS NCEP ATPIII criteria, without CVD or diabetes mellitus, but most of the other studies were focused on advanced stages of MetS.

We observed that GPx was associated with levels of TMPs, EMPs, and PMPs in the MetS subjects. In agreement with our findings, Helal et al found that GPx was positively correlated with TMP and PMP levels in subjects with MetS. ${ }^{23}$ This finding supports increased cellular antioxidant activity, which was probably upregulated in the presence of augmented oxidative stress.

In this study, MetS parameters correlated positively with the GPx enzyme. Increased ROS production could be due to high glucose levels leading to protein glycation and glucose autoxidation..$^{42}$ Moreover, increased production of free fatty acids induced by dyslipidemia enhances the production of ROS. ${ }^{43}$ Also, accumulation of TGs and LDL triggers increased oxidative stress, which plays an important role in the pathogenesis of atherosclerosis. ${ }^{44}$ However, HDL has antioxidant properties. Oxidative changes in lipoprotein metabolism thus play an important role in the development of CVD. ${ }^{45}$

It has been reported that elevated PAI1 levels are an important contributor to CVD through reducing blood fibrinolytic activity. Our findings concerning elevated levels of the PAI1-TPA complex among MetS patients were in line with previous studies. ${ }^{17,46}$ The overproduction of PAI1 in MetS patients is a true component of the syndrome. ${ }^{47}$ Mounting evidence based on previous studies indicates that plasma TPA and PAI1 are regulated in part by the renin-angiotensin system and that polymorphisms in genes from the reninangiotensin-bradykinin system are associated with PAI1 and TPA levels. ${ }^{48,49}$ In this study, multivariate-regression results revealed that PAI1/TPA levels were influenced by components of MetS, except fasting hyperglycemia level. As such, fibrinolysis was found to be severely influenced by MetS. Also, Huotari et al found that increased circulating PAI1 is strongly linked to MetS and is an independent component of the syndrome ${ }^{50}$ Therefore, these results support the hypothesis that many metabolic disorders are associated with elevation of plasma PAI1 and reduction of TPA activities, since PAI1 is a multifunctional protein.

One of the limitations of our study is its cross-sectional nature. We were not able to perform any follow-up. Additionally, due to a lack of funding, we measured GPx as the only oxidative stress-related variable. 


\section{Conclusion}

Elevated circulating MPs are associated with MetS abnormalities, oxidative stress and coagulation factors and may act as early predictor of metabolic syndrome with risk of CVD. Further studies are required to determine the validity of using MPs as biomarkers for progressive metabolic abnormalities and as a target for treatment. We believe that evaluating changes in totals of a specific population of circulating MPs in response to pharmacological treatment could indicate the efficacy of a treatment or disease regression. Moreover, modulation of the level of MPs by drug treatment could reduce the deleterious effects of MPs. It would be of interest to assess the relationship between BP-lowering medications and EMPs. Drugs used for the treatment of hypertension could modify EMP release by reducing shear stress. Further analyses of MP detection and quantification for each patient could allow personalized therapy.

\section{Abbreviations}

BMI, body-mass index; CVD, cardiovascular disease; DBP, diastolic blood pressure; EMPs, endothelium-derived microparticles; FBG, fasting blood glucose; GPx, glutathione peroxidase; HDL, high-density lipoprotein; LDL, low-density lipoprotein; MetS, metabolic syndrome; MPs, microparticles; PMPs, platelet-derived microparticles; SBP, systolic blood pressure; TG, triglyceride; TMPs, total microparticles; WC, waist circumference.

\section{Disclosure}

The authors report no conflicts of interest in this work.

\section{References}

1. Berezin AE, Kremzer AA, Samura TA, Berezina TA, Kruzliak P. Immune phenotypes of endothelial-derived microparticles in dysmetabolic patients. J Proteomics Bioinform. 2015;8(3):60.

2. Eckel RH, Grundy SM, Zimmet PZ. The metabolic syndrome. Lancet. 2005;365(9468):1415-1428.

3. Wilson PWF, D'Agostino RB, Parise H, Sullivan L, Meigs JB. Metabolic syndrome as a precursor of cardiovascular disease and type 2 diabetes mellitus. Circulation. 2005;112(20):3066-3072.

4. Burger D, Schock S, Thompson CS, Montezano AC, Hakim AM, Touyz RM. Microparticles: biomarkers and beyond. Clin Sci. 2013;124(7):423-441.

5. Xu MD, Wu XZ, Zhou Y, Xue Y, Zhang KQ. Proteomic characteristics of circulating microparticles in patients with newly-diagnosed type 2 diabetes. Am J Transl Res. 2016;8(1):209.

6. Zaghloul A, Al-Bukhari TAMA, Al-Pakistani HA, et al. Soluble endothelial protein $\mathrm{C}$ receptor and high sensitivity $\mathrm{C}$ reactive protein levels as markers of endothelial dysfunction in patients with type 1 and type 2 diabetes mellitus: their role in the prediction of vascular complications. Diabetes Res Clin Pract. 2014;106(3):597-604.
7. Ahmadi A, Leipsic J, Feuchtner G, et al. Is metabolic syndrome predictive of prevalence, extent, and risk of coronary artery disease beyond its components? Results from the multinational coronary CT angiography evaluation for clinical outcome: an international multicenter registry (confirm). PLoS One. 2015;10(3):e0118998.

8. Barteneva NS, Fasler-Kan E, Bernimoulin M, et al. Circulating microparticles: square the circle. BMC Cell Biol. 2013;14(1):23.

9. Ardoin SP, Shanahan JC, Pisetsky DS. The role of microparticles in inflammation and thrombosis. Scand J Immunol. 2007;66(2-3):159-165.

10. Milioli M, Ibáñez-Vea M, Sidoli S, Palmisano G, Careri M, Larsen MR. Quantitative proteomics analysis of platelet-derived microparticles reveals distinct protein signatures when stimulated by different physiological agonists. J Proteomics. 2015;121:56-66.

11. Roberts CK, Sindhu KK. Oxidative stress and metabolic syndrome. Life Sci. 2009;84(21-22):705-712.

12. Bellanti F, Matteo M, Rollo T, et al. Sex hormones modulate circulating antioxidant enzymes: impact of estrogen therapy. Redox Biol. 2013;1(1):340-346.

13. Samsam-Shariat SZ, Bolhasani M, Sarrafzadegan N, Najafi S, Asgary S. Relationship between blood peroxidases activity and visfatin levels in metabolic syndrome patients. ARYA Atheroscler. 2014;10(4):218-226.

14. Dellas C, Loskutoff DJ. Historical analysis of PAI-1 from its discovery to its potential role in cell motility and disease. Thromb Haemost. 2005;93(4):631-640.

15. Kohler HP, Grant PJ. Plasminogen-activator inhibitor type 1 and coronary artery disease. $N$ Engl J Med. 2000;342(24):1792-1801.

16. Lijnen HR. Pleiotropic functions of plasminogen activator inhibitor-1. J Thromb Haemost. 2005;3(1):35-45.

17. Anand SS, Yi Q, Gerstein H, et al. Relationship of metabolic syndrome and fibrinolytic dysfunction to cardiovascular disease. Circulation. 2003;108(4):420-425.

18. Berezin AE, Kremzer AA, Martovitskaya YV, Samura TA, Berezina TA. The predictive role of circulating microparticles in patients with chronic heart failure. BBA Clin. 2015;3:18-24.

19. Thomas GN, Ho SY, Janus ED, et al. The US national cholesterol education programme adult treatment panel III (NCEP ATP III) prevalence of the metabolic syndrome in a Chinese population. Diabetes Res Clin Pract. 2005;67(3):251-257.

20. Gierach M, Gierach J, Ewertowska M, Arndt A, Junik R. Correlation between body mass index and waist circumference in patients with metabolic syndrome. ISRN Endocrinol. 2014;2014(10):1-6.

21. Hekmatdoost A, Mirmiran P, Hosseini-Esfahani F, Azizi F. Dietary fatty acid composition and metabolic syndrome in Tehranian adults. Nutrition. 2011;27(10):1002-1007.

22. Nasreddine L, Ouaijan K, Mansour M, Adra N, Sinno D, Hwalla N. Metabolic syndrome and insulin resistance in obese prepubertal children in Lebanon: a primary health concern. Ann Nutr Metab. 2010;57(2):135-142.

23. Helal O, Defoort C, Robert S, et al. Increased levels of microparticles originating from endothelial cells, platelets and erythrocytes in subjects with metabolic syndrome: relationship with oxidative stress. Nutr Metab Cardiovasc Dis. 2011;21(9):665-671.

24. Zaki ME, El-Bassyouni HT, El-Gammal M, Kamal S. Indicators of the metabolic syndrome in obese adolescents. Arch Med Sci. 2015;11(1):92-98.

25. Johnson WD, Kroon JJM, Greenway FL, Bouchard C, Ryan D, Katzmarzyk PT. Prevalence of risk factors for metabolic syndrome in adolescents: National Health and Nutrition Examination Survey (NHANES), 2001-2006. Arch Pediatr Adolesc Med. 2009;163(4):371-377.

26. Agirbasli M, Agaoglu NB, Ergonul O, et al. Comparison of anthropometric indices in predicting metabolic syndrome components in children. Metab Syndr Relat Disord. 2011;9(6):453-459.

27. Eshtiaghi R, Keihani S, Hosseinpanah F, Barzin M, Azizi F. Natural course of metabolically healthy abdominal obese adults after 10 years of follow-up: the Tehran lipid and glucose study. Int $J$ Obes. 2015;39(3):514-519. 
28. Agouni A, Lagrue-Lak-Hal AH, Ducluzeau PH, et al. Endothelial dysfunction caused by circulating microparticles from patients with metabolic syndrome. Am J Pathol. 2008;173(4):1210-1219.

29. Ueba T, Haze T, Sugiyama M, et al. Level, distribution and correlates of platelet-derived microparticles in healthy individuals with special reference to the metabolic syndrome. Thromb Haemost. 2008;100(2):280-285.

30. Arteaga RB, Chirinos JA, Soriano AO, et al. Endothelial microparticles and platelet and leukocyte activation in patients with the metabolic syndrome. Am J Cardiol. 2006;98(1):70-74.

31. Hanzu FA, Palomo M, Kalko SG, et al. Translational evidence of endothelial damage in obese individuals: Inflammatory and prothrombotic responses. J Thromb Haemost. 2011;9(6):1236-1245.

32. Aye M, Sazali M. Waist circumference and BMI cut-off points to predict risk factors for metabolic syndrome among outpatients in a district hospital. Singapore Med J. 2012;53(8):545.

33. Bouguerra R, Alberti H, Smida H, et al. Waist circumference cut-off points for identification of abdominal obesity among the Tunisian adult population. Diabetes Obes Metab. 2007;9(6):859-868.

34. Goichot B, Grunebaum L, Desprez D, et al. Circulating procoagulant microparticles in obesity. Diabetes Metab. 2006;32(1):82-85.

35. Stepanian A, Bourguignat L, Hennou S, et al. Microparticle increase in severe obesity: not related to metabolic syndrome and unchanged after massive weight loss. Obesity. 2013;21(11):2236-2243.

36. Diamant M, Nieuwland R, Pablo RF, Sturk A, Smit JWA, Radder JK. Elevated numbers of tissue-factor exposing microparticles correlate with components of the metabolic syndrome in uncomplicated type 2 diabetes mellitus. Circulation. 2002;106(19):2442-2447.

37. Chironi GN, Boulanger CM, Simon A, Dignat-George F, Freyssinet J-M, Tedgui A. Endothelial microparticles in diseases. Cell Tissue Res. 2009;335(1):143-151.

38. Shantsila E. Endothelial microparticles: a universal marker of vascular health? J Hum Hypertens. 2009;23(5):359-361.

39. Erdeve O, Siklar Z, Kocaturk PA, Dallar Y, Kavas GO. Antioxidant superoxide dismutase activity in obese children. Biol Trace Elem Res. 2004;98(3):219-228
40. Armutcu F, Ataymen M, Atmaca H, Gurel A. Oxidative stress markers, $\mathrm{C}$-reactive protein and heat shock protein 70 levels in subjects with metabolic syndrome. Clin Chem Lab Med. 2008;46(6):785-790.

41. Ozata M, Mergen M, Oktenli C, et al. Increased oxidative stress and hypozincemia in male obesity. Clin Biochem. 2002;35(8):627-631.

42. Nowotny K, Jung T, Höhn A, Weber D, Grune T. Advanced glycation end products and oxidative stress in type 2 diabetes mellitus. Biomolecules. 2015;5(1):194-222.

43. De Oliveira J, Hort MA, Moreira ELG, et al. Positive correlation between elevated plasma cholesterol levels and cognitive impairments in LDL receptor knockout mice: relevance of cortico-cerebral mitochondrial dysfunction and oxidative stress. Neuroscience. 2011;197:99-106.

44. Welty FK. How do elevated triglycerides and low HDL-cholesterol affect inflammation and atherothrombosis? Curr Cardiol Rep. 2013;15(9):400.

45. Demircan N, Gurel A, Armutcu F, Unalacak M, Aktunc E, Atmaca H The evaluation of serum cystatin $\mathrm{C}$, malondialdehyde, and total antioxidant status in patients with metabolic syndrome. Med Sci Monit. 2008;14(2):CR97-CR101.

46. Coffey CS, Asselbergs FW, Hebert PR, et al. The association of the metabolic syndrome with PAI-1 and t-PA levels. Cardiol Res Pract. 2011;2011(4):1-8.

47. Alessi MC, Juhan-Vague I. PAI-1 and the metabolic syndrome: links, causes, and consequences. Arterioscler Thromb Vasc Biol. 2006;26(10):2200-2207.

48. Asselbergs FW, Williams SM, Hebert PR, et al. Epistatic effects of polymorphisms in genes from the renin-angiotensin, bradykinin, and fibrinolytic systems on plasma t-PA and PAI-1 levels. Genomics. 2007;89(3):362-369.

49. Kim DK, Kim JW, Kim S, et al. Polymorphism of angiotensin converting enzyme gene is associated with circulating levels of plasminogen activator inhibitor-1. Arterioscler Thromb Vasc Biol. 1997;17(11): 3242-3247.

50. Huotari A, Lehto SM, Niskanen L, et al. Increased serum PAI-1 levels in subjects with metabolic syndrome and long-term adverse mental symptoms: a population-based study. Cardiovasc Psychiatry Neurol. 2010;2010(1):1-7.

\section{Publish your work in this journal}

Diabetes, Metabolic Syndrome and Obesity: Targets and Therapy is an international, peer-reviewed open-access journal committed to the rapid publication of the latest laboratory and clinical findings in the fields of diabetes, metabolic syndrome and obesity research Original research, review, case reports, hypothesis formation, expert opinion and commentaries are all considered for publication. The manuscript management system is completely online and includes a very quick and fair peer-review system, which is all easy to use. Visit http://www.dovepress.com/testimonials.php to read real quotes from published authors.

Submit your manuscript here: https://www.dovepress.com/diabetes-metabolic-syndrome-and-obesity-targets-and-therapy-journal 